\title{
E3 Ubiquitin Ligase March I Facilitates OX40L Expression in Allergen-Stimulated Dendritic Cells Through Mediating the Ubiquitination of HDACI I
}

\author{
Xinxing Zhang* \\ Zhichao Sun* \\ Suyu Guo \\ Jiahui Zhang \\ Wenjing Gu \\ Zhengrong Chen \\ Li Huang
}

Department of Pediatric Pulmonology, Children's Hospital of Soochow University, Suzhou, Jiangsu, 215003,

People's Republic of China

*These authors contributed equally to this work
Correspondence: Zhengrong Chen; Li Huang

$\mathrm{Tel} / \mathrm{Fax}+86-05 / 2-80693588$

Email chen_zheng_rong@163.com; szdalv@।63.com
Background: It was demonstrated that membrane-associated RING-CH 1 (March 1) might play an important role in the pathogenesis of asthma.

Methods: The levels of mRNA and protein were measured by qRT-PCR and Western blot, respectively. Immunofluorescence assay was used to determine whether March1 co-locates with HDAC11. Co-immunoprecipitation was performed to examine the combination of proteins. Moreover, luciferase assay was used to measure the promoter activity of genes.

Results: The mRNA and protein levels of both March1 and OX40 ligand (OX40L) were increased in the dendritic cells (DCs) from asthmatic children and asthmatic animals. Histone deacetylase 11 (HDAC11) protein was decreased in the DCs from asthmatic children and asthmatic model. Increasing of March1 or decreasing of March1 only affect the expression of HDAC11 in protein level. Besides, increasing of HDAC11 could inhibit OX40L expression, and decreasing of HDAC11 promoted OX40L expression in house dust mites (HDMs)-treated DCs. Increasing of HDAC11 notably reversed the promotion of March1 to OX40L expression. Our data further proved that March1 reduced the protein level of HDAC11 through inducing ubiquitination and degradation. HDAC11 combined with krüppel-like factor 4 (KLF4) to decrease the activity of OX40L gene promoter, thus to downregulate the level of OX40L.

Conclusion: Overall, our data showed that HDAC11 promoted KLF4-dependent OX40L decreasing. However, March1 promoted OX40L expression through enhancing the ubiquitination and degradation of HDAC11 and subsequent blocking the inhibition of HDAC11 to OX40L.

Keywords: asthma, march1 regulating OX40 ligand, ubiquitination, histone deacetylase 11, OX40 ligand

\section{Introduction}

Asthma is an acute respiratory disorder characterised by the chronic inflammation of airway. In clinical, asthmatic patients have wheezing sound in breathing, cough, severe shortness of breath, and tightness in chest and other symptoms. ${ }^{1,2}$ Asthma exacerbation is one of the top three reasons for hospitalization in children. The children with atopic asthma have a slow decline in lung function over time. ${ }^{3,4}$ As the report of the Center for Disease Control and Prevention in 2016, the prevalence of asthma is $9.6 \%$ in the children aged 5 to 11 years and $10.5 \%$ in the children aged 12 to 17 years globally. ${ }^{5}$ Asthma in children is a huge global health burden, and the improvement in diagnosis and management has been unsatisfactory. Hence, it is necessary to explore the pathogenesis of asthma. ${ }^{3,6,7}$ Allergic asthma can be triggered by a variety of allergens, such as house dust mites (HDMs). ${ }^{8}$ 
Ovalbumin (OVA) is a common allergen which was used to establish mouse asthmatic model. ${ }^{9}$ At present, although a great advance has been made in therapy, asthma still is a high-risk condition.

Inflammation is an important pathological characteristic of asthma. It was reported that numerous immune cells infiltrated into the airways of asthmatic patients, such as lymphocytes, degranulated mast cells and eosinophils. ${ }^{10}$ Malmström et al proved that the numbers of plasmacytoid dendritic cells (DCs), eosinophils and macrophages in the lung tissues of asthmatic children significantly more than that in the lung tissues of healthy children. ${ }^{11}$ Moreover, some studies demonstrated that the airway DCs are activated, and then the activated DCs involve in airway epithelial barrier dysfunction and airway inflammation in asthma. ${ }^{12}$ Existing evidences have indicated the important role of DCs in the occurrence and development of asthma. Ubiquitination, phosphorylation and sumoylation are the important regulatory mechanisms of gene expression, and participate in the development of many disorders including asthma. Ubiquitin-proteasome pathway is composed of three enzymes that activate (E1), conjugate (E2) and ligate (E3). ${ }^{13}$ Membrane-associated RING-CH 1 (March 1) belongs to E3 ubiquitin ligases, and it mostly expresses in spleen. ${ }^{14,15}$ Airway hyperresponsiveness and the levels of inflammatory cytokines are lower in the lung tissues of OVA-induced asthmatic March $1^{-/}$mice than wide-type mice, suggesting March1 may play a crucial role in the development of asthma. ${ }^{16}$

Acetylation also participates in the regulation of asthma development. Histone deacetylase11 (HDAC11) is the newest member of the histone deacetylase (HDAC) family and the only class IV histone deacetylase. ${ }^{17}$ A previous study indicated that the members of HDAC family, including $\mathrm{HDAC} 7, \mathrm{HDAC} 9, \mathrm{HDAC} 10$, and HDAC11, are abnormally expressed in the allergenspecific memory $\mathrm{T}$ cells and involve in the development of asthma. ${ }^{18}$ Furthermore, in our present study, we explored the molecular mechanism of March1 regulating OX40 ligand (OX40L) expression in HDM-stimulated DCs. OX40L is a member of the TNF superfamily and was first identified as gp34. ${ }^{19}$ It was revealed that OX40L is highly expressed in the serum of asthmatic patients compared to healthy ones. Moreover, OX40L have been proved to regulate the differentiation of helper $\mathrm{T}$ cells in asthma via targeting MAPK and PI3K/AKT signaling pathways. $^{20}$ The existing studies indicated that exploring the regulatory mechanism of $\mathrm{OX} 40 \mathrm{~L}$ expression might provide a new target for the treatment of asthma. Here, our data proved a new pathogenesis of asthma, and might provide a novel idea or target for the treatment of the disease.

\section{Materials and Methods Clinical Samples}

A total of 20 bronchial asthmatic children and 20 healthy children were recruited from our hospital between December 2018 and December 2019. Peripheral blood samples were collected from the asthmatic children and healthy children. DCs were isolated from above samples in accordance with the previous study. ${ }^{21}$ Next, qRT-PCR and Western blot were respectively used to detect the mRNA and protein levels of March1, HDAC11 and OX40L in DCs from asthmatic children (Asthma group) and healthy children (HC group). This study was approved by the ethics committee of the Children's Hospital of Soochow University (2018LW096). The informed consents have been written by the family members of asthmatic children and healthy children before our research. The children in healthy control (HC) group have no history of chronic respiratory disease. And this study was conducted in accordance with the Declaration of Helsinki.

\section{Mouse Asthmatic Model}

A total of $20 \mathrm{BALB} / \mathrm{C}$ mice (gender, male; aged, 6-8 weeks; weight, 20-30 g) were purchased from Charles River (Beijing, China). All mice were housed in a suitable environment with $12 \mathrm{~h}$ dark/light cycle, temperature in $25^{\circ} \mathrm{C}$, and enough food and water. One week after adaptation, the mice were randomly divided into two groups: control and OVA. All animal experiments were approved by the Animal Ethics Committee of Soochow University (SUDA20200510A02). Meantime, all experiments were carried out strictly according to the request of the Recommended Guideline for the Care and Use of Laboratory Animals formulated by the Chinese Council on Animal Research.

The mice in OVA group were intraperitoneally injected with $0.2 \mathrm{~mL}$ of normal saline containing $0.2 \mathrm{mg}$ OVA (Sangon Biotech Co., Ltd, Shanghai, China) and $1 \mathrm{mg}$ aluminum hydroxide (Beijing Solarbio Science \& Technology Co., Ltd, China) at 1, 7, and 14 day. Next step, at 21, 22, 23, 24, and 25 day, the mice were challenged with 5\% OVA for 30min daily. At 24 hours after the last challenge, peripheral bloods of the mice were 
collected, and then were used for qRT-PCR and Western blot analysis. The mice in control group were sensitization and challenge with normal saline.

\section{Cell Culture and Treatment}

Bone marrow cells were isolated from normal mice, and were used to generate DCs as previous study. ${ }^{22}$ For asthma simulation in vivo, $100 \mu \mathrm{g} / \mathrm{mL}$ of HDM was utilized to treat the DCs generated from bone marrow cells of normal mice (HDM group). The PBS-treated DCs served as control group. At 24 hours after HDM stimulation, qRT-PCR and Western blot were carried out to examine the mRNA and protein levels of March1, HDAC11 and OX40L, respectively.

All cells were cultured at $37^{\circ} \mathrm{C}$ in an incubator with $5 \%$ $\mathrm{CO}_{2}$. The cells were grown in RPMI-1640 medium (Gibco-BRL, Carlsbad, CA, USA) supplemented with $10 \%$ fetal bovine serum (FBS; Gibco-BRL). In our study, $50 \mu \mathrm{g} / \mathrm{mL}$ cycloheximide (CHX; Sigma-Aldrich) was used to inhibit protein synthesis, and $10 \mu \mathrm{g} / \mathrm{mL}$ MG132 (SigmaAldrich) was used to inhibit ubiquitin- proteasome pathway. The lentivirus vectors packaged with pcDNA3.1-March1 (LV-March1) or empty vector (LVmock), March1 shRNA (shMarch1) or shRNA negative control (LV-shRNA), pcDNA3.1-HDAC11 (LV-HDAC11) , and HDAC11 shRNA (LV-shHDAC11) or its negative control (LV-shRNA) were conducted by GeneChem (Shanghai, China), and were used to increase or decrease the expression of target molecules. In addition, KLF4 siRNA (si-KLF4) and its negative control (siRNA) were transfected into DCs using Lipofectamine ${ }^{\circledR} 3000$ (Invitrogen; Thermo Fisher Scientific, Inc.) according to the manufacture's introduction. After 24 hours of lentivirus infection and transfection, qRT-PCR, Western blotting assay and other experiments were performed.

\section{QRT-PCR}

Total RNA was isolated from DCs using TRIzol reagent (Invitrogen, Carlsbad, CA, USA). Then, 50 ng of RNA was reverse transcribed into cDNA according to the PrimeScript RT reagent Kit (Invitrogen). Next, qPCR was carried out to detect the relative expression of March1, HDAC11, and OX40L on a StepOnePlus realtime PCR system (Applied Biosystems, Inc., Foster City, CA) using the SYBR Green real-time PCR Master Mix (Invitrogen). The relative expression levels of genes were analyzed as the method of $2^{-\Delta \Delta \mathrm{Ct}}$, and normalize to
$G A P D H$. All experiments were carried out in accordance with the manufacture's introduction of reagent.

\section{Western Blot}

Total protein was isolated from DCs using RIPA lysis buffer (Solarbio, Beijing, China). Then, $25 \mu \mathrm{g}$ of protein was separated on the $12 \%$ SDS-PAGE, and subsequent was transferred into PVDF membranes (Sigma-Aldrich). Next, the membranes were blocked with $5 \%$ non-fat milk for 1 hour at room temperature, primary antibodies against March1 (1:2000, Abcam, Cambridge, MA), HDAC11 (1:1000, Abcam), OX40L (1:1000, Abcam), and KLF4 (1:2000, Abcam) overnight at $4^{\circ} \mathrm{C}$ followed by secondary antibodies (1:3000, Abcam) for 1 hour at room temperature. At last, an ECL kit was used to display the protein bands, and Image $\mathrm{J}$ software was used to analyze the relative expression levels of proteins. GAPDH served as the internal reference of target proteins.

\section{Co-Immunoprecipitation (Co-IP) Assay}

The combination of HDAC11 and March1 or KLF4 were ensured by Co-IP assay. The eukaryotic expression plasmids of March1 with flag tag (Flag-March1), and the plasmids of HDAC11 with myc tag (Myc-HDAC11) were constructed. Flag-March1 and Myc-HDAC11 plasmids were transfected into HERK293T cells. At 24 hours after transfection, the cells were lysed for Co-IP assay. Besides, at 24 hours after HDM stimulation, the DCs were lysed for Co-IP assay. The cell supernatants were incubated with anti-March1, anti-HDAC11 or anti-KLF4 monoclonal antibodies for 1 hour at $4^{\circ} \mathrm{C}$. Then, $30 \mu \mathrm{L}$ of Protein $\mathrm{G}$ beads were maintained with the cell supernatants overnight at $4^{\circ} \mathrm{C}$. After that, the beads were washed with lysis buffer for three times, and then Western blot was performed to detect HDAC11 and March1 expression.

\section{Analysis of HDACI I Ubiquitination}

HA-UB and Flag-HDAC11 plasmids were co-transfected into DCs. Meantime, the cells were co-transfected with or without Myc-March1 plasmids. At 24 hours after cell transfection, DCs were lysed, and then partly cell lysates were used as input. The rest of cell lysates were used in immunoprecipitation assay.

\section{Immunofluorescence Assay}

For immunofluorescence assay, DCs were planted into 24well plates at a density of $1 \times 10^{5}$ cells/well. The plasmids expressing March1 and the plasmids expressing HDAC11 
were transfected into HDM-stimulated DCs. At 24 hours after transfection, the cells were fixed with $4 \%$ paraformaldehyde for $30 \mathrm{~min}$ at $4{ }^{\circ} \mathrm{C}$. Then, the cells were blocked with $5 \% \mathrm{BSA}$ blocking buffer (Solarbio) for 1 hour at room temperature, and were maintained with primary antibodies against March1 (1:200) and HDAC11 (1:200) overnight at $4^{\circ} \mathrm{C}$. After that, the cells were stained with fluorescein-labelled secondary antibody (1:50; Dianova, Hamburg, Germany) for 1 hour at room temperature in the dark. Next, 4,6-diamidino-2-phenylindole was used to stain the nuclei of DCs for $5 \mathrm{~min}$ at room temperature in the dark. At last, March1-positive and HDAC11-positive DCs were observed under the confocal laser scanning microscopy (Olympus, Tokyo, Japan).

\section{Luciferase Assay}

Firstly, constructing the luciferase reporter vectors containing wild-type (WT) or mutation (MUT) gene sequences of $O X 40 L$ promoter. Then, due to the different aims of our studies, the recombinant luciferase reporter vectors were co-transfected with the plasmids expressing KLF4 and empty vector, the plasmids expressing KLF4 siRNA and siRNA negative control, and LV-HDAC11 and LV-shHDAC11 into HDM-treated DCs cells. At 24 hours after cell transfection, luciferase assay was carried out to detect the promoter activity of OX40L.

\section{Statistical Analysis}

GraphPad Prism 7 software was used for data analysis. Our data were displayed as mean \pm standard deviation (SD). All experiments were independently repeated at least three times. The significant difference between two independent groups was ensured by Student's $t$-test, and one-way analysis of variance (ANOVA) followed by post hoc Dunnet's multiple-comparison test was used for the analysis of multiple groups. A value of $P$ lower than 0.05 was recognized as statistically significant.

\section{Results}

MarchI and OX40L Were Increased, While HDACI I Protein Was Decreased in Asthma

In this study, our data indicated that the expression of March1 mRNA (Figure 1A) and OX40L mRNA (Figure 1C) in the DCs from asthmatic patients was notably higher than those in the DCs from healthy volunteers. However, the level of HDAC11 mRNA in the DCs from asthmatic patients had no significant difference with in the
DCs from healthy volunteers (Figure 1B). We also examined the expression levels of March1 protein, HDAC11 protein and OX40L protein in the DCs from asthmatic patients and healthy volunteers. Our results showed that the expression of March1 and OX40L were higher, whereas HDAC11 was lower in the DCs from asthmatic patients than those in the DCs from healthy volunteers (Figure 1D). Next step, we further measured the expression of March1, OX40L, and HDAC11 in the DCs from asthmatic mice and in the HDM-stimulated DCs. Our data indicated that the expression of March1 mRNA was markedly increased in the DCs from asthmatic mice (Figure 1E) and HDM-stimulated DCs (Figure 1I). Also, the expression of OX40L mRNA was notably increased in the DCs from asthmatic mice (Figure 1G) and HDM-stimulated DCs (Figure 1K). However, the expression of HDAC11 mRNA in the DCs from asthmatic mice and in the HDMstimulated DCs had no significant difference with that in the DCs from normal mice and control DCs, respectively (Figure 1F and J). In addition, as shown in Figure 1H and $\mathrm{L}$, the expression of March1 and OX40L proteins was increased, whereas HDAC11 was decreased in the DCs from asthmatic mice and HDM-stimulated DCs. Overall, the gene and protein levels of March1 and OX40L were increased in asthma, while the only protein level of HDAC11 was decreased in asthma.

\section{March I Inhibited HDACII, While Promoted OX40L Expression}

To explore the effect of March1 on the expression of HDAC11 and OX40L in HDM-stimulated DCs, we established the March1 expressing lentivirus vector, and obtained March1 shRNA. As shown in Figure 2A and B, the expression of March1 mRNA and March1 protein in DCs was significantly upregulated by LV-March1 infection. In addition, the expression of March1 mRNA and March1 protein in DCs was notably downregulated by LV-shMarch1-1 and LV-shMarch1 -2 treatment (Figure 2C and D). LV-shMarch1-2 was used in following studies to interfere the expression of March1 in DCs. Interestingly, our results indicated that increasing or decreasing of March1 had no effects on the expression of HDAC11 mRNA in HDM-treated DCs (Figure 2E). The level of OX40L mRNA in HDM-treated DCs was notably higher than that in control DCs. The expression of OX40L mRNA in HDM-stimulated DCs was promoted by LVMarch1 treatment, and was inhibited by LV-shMarch1 treatment (Figure 2F). Consistently, the results of Western blotting 


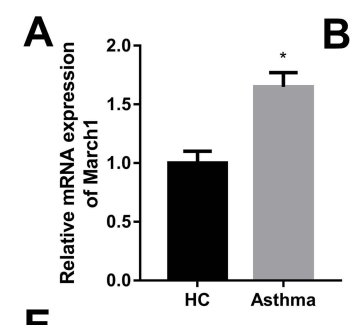

E

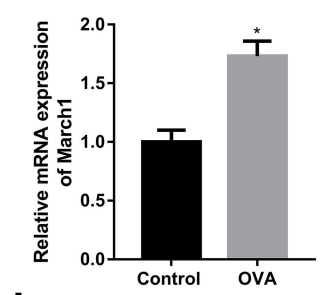

I

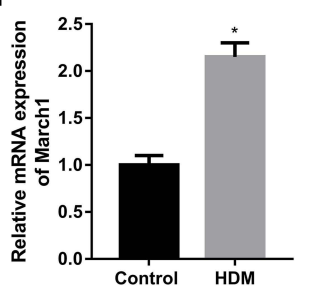

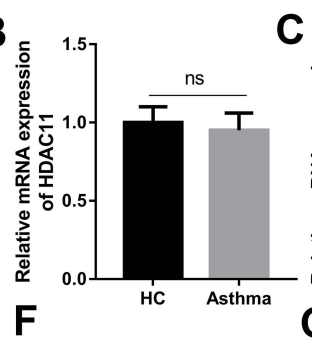
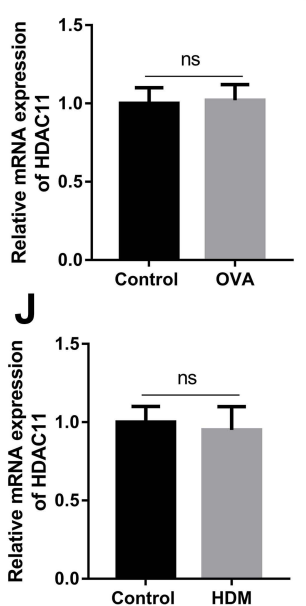

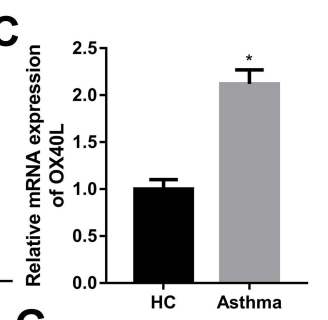

G
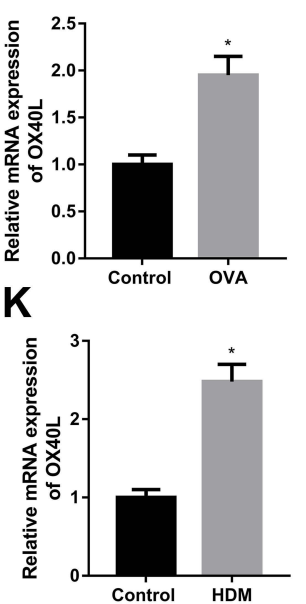
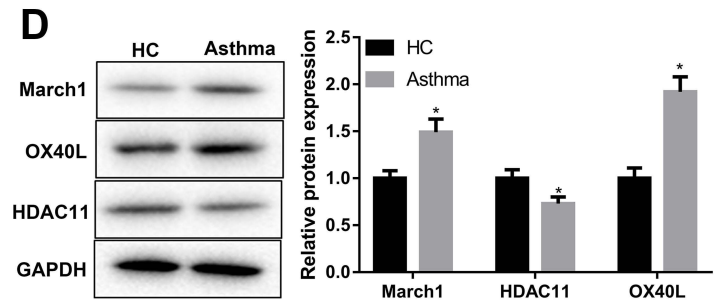

H
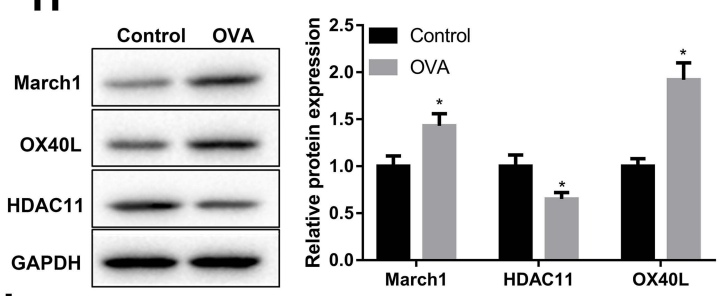

$\mathbf{L}$
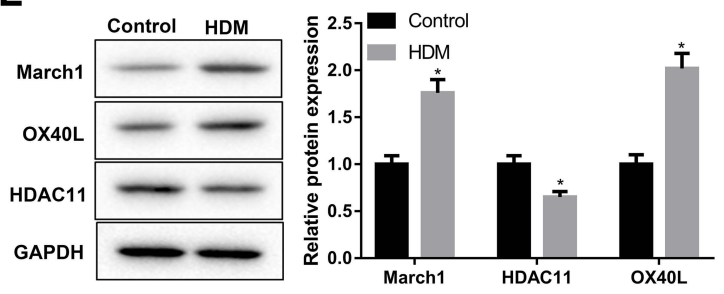

Figure I Detection of the expression of MarchI, HDACII and OX40L in asthma. Peripheral blood samples were obtained from asthmatic patients and healthy volunteers, and then DCs were isolated from the serum. Next, (A-C) qRT-PCR was performed to detect the levels of March I mRNA, HDACII mRNA and OX40L mRNA in DCs. (D) Western blot was carried out to detect the expression of MarchI, OX40L and HDACII in DCs. $\mathrm{n}=20$. $* \mathrm{p}<0.05$ compared with HC group, and ns was no significant difference. Peripheral blood samples were obtained from asthmatic mice and normal mice, and then DCs were isolated from the serum. Next, (E-G) qRT-PCR was performed to detect the levels of MarchI mRNA, HDACII mRNA and OX40L mRNA in DCs. (H) Western blot was carried out to detect the expression of MarchI, OX40L and HDACII in DCs. $n=10$. $* P<0.05$ compared with Control group, and ns was no significant difference. After that, DCs were isolated from the marrow cells of normal mice, and were stimulated with HDM. (I-K) qRT-PCR was performed to detect the levels of March I mRNA, HDACII mRNA and OX40L mRNA in DCs. (L) Western blot was carried out to detect the expression of MarchI, OX40L and HDACII in DCs. $n=3$. *P $<0.05$ compared with Control group, and ns was no significant difference.

assay showed that the level of OX40L protein also was increased in HDM-stimulated DCs. LV-March1 treatment further facilitated the expression of OX40L protein in HDMstimulated DCs, while LV-shMarch1 treatment downregulated the expression of OX40L protein. Importantly, our data revealed that the protein level of HDAC11 in HDM-treated DCs was notably lower than that in control DCs. Overexpression of March1 suppressed, and silencing of March1 increased the expression of HDAC11 in protein level in HDM-stimulated DCs (Figure 2G and H). Overall, in HDM-treated DCs, March1 positively regulated the expression of OX40L mRNA and protein, and negatively regulated the expression of HDAC11 protein.

\section{March I Promoted OX40L Expression via Reducing the Protein Level of HDACI I}

To investigate the effect of HDAC11 on OX40L expression, we also obtained the lentivirus vector expressing HDAC11 or HDAC11 shRNA. Our data showed that the expression of HDAC11 mRNA and protein in DCs was significantly upregulated by LV-HDAC11 treatment (Figure 3A and B). The shRNA of HDAC11 significantly suppressed HDAC11 mRNA and protein expression in DCs (Figure 3C and D). HDAC11 shRNA-2 was used in following studies. Furthermore, the results indicated that HDAC11 increasing in HDM-stimulated DCs suppressed OX40L mRNA and protein expression, while silencing of HDAC11 promoted OX40L mRNA and protein expression (Figure $3 \mathrm{E}$ and $\mathrm{F}$ ). In addition, in HDM-treated DCs, increasing of March1 notably upregulated the gene and protein levels of OX40L, which were partly reversed by HDAC11 increasing (Figure $3 \mathrm{G}$ and $\mathrm{H}$ ). In summary, our data showed that HDAC11 inhibited OX40L expression and reversed the promontory effect of March1 on OX40L expression.

\section{March I Facilitated the Ubiquitination and Degradation of HDACI I}

March1 is an E3 ubiquitin ligase. Hence, we next explored whether March1 affects OX40L expression via promoting the ubiquitination of HDAC11. Here, we detected the 

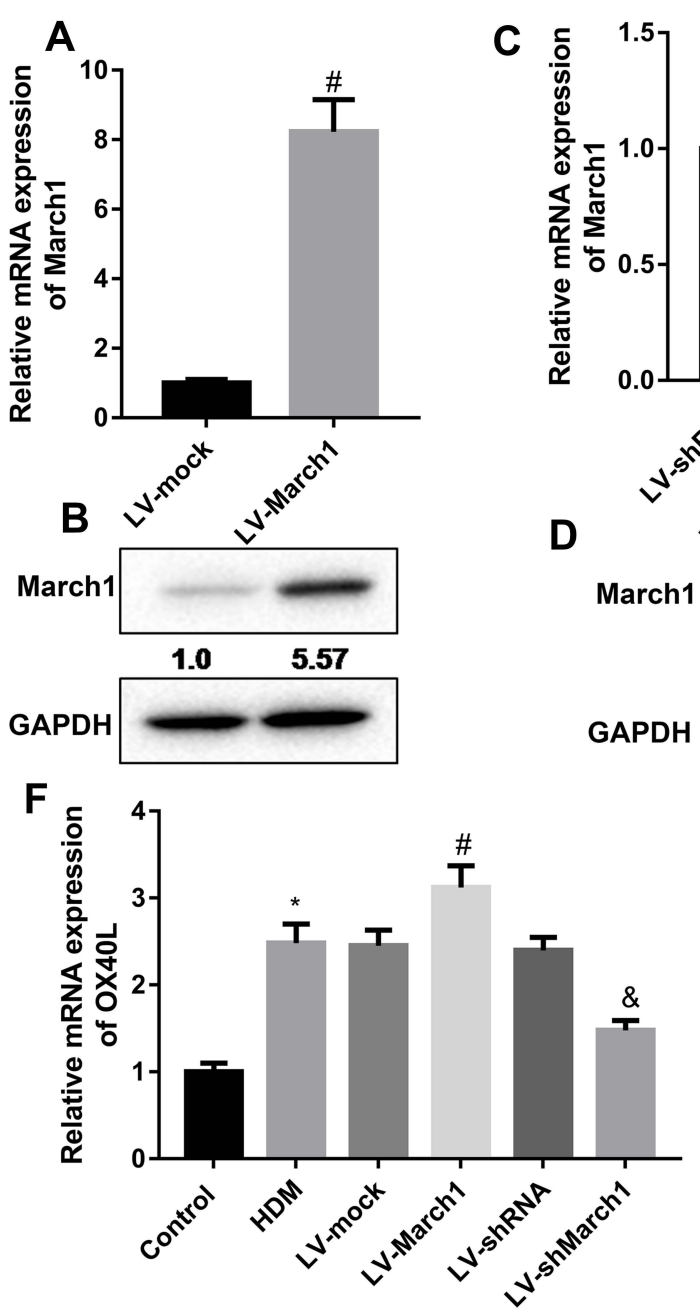
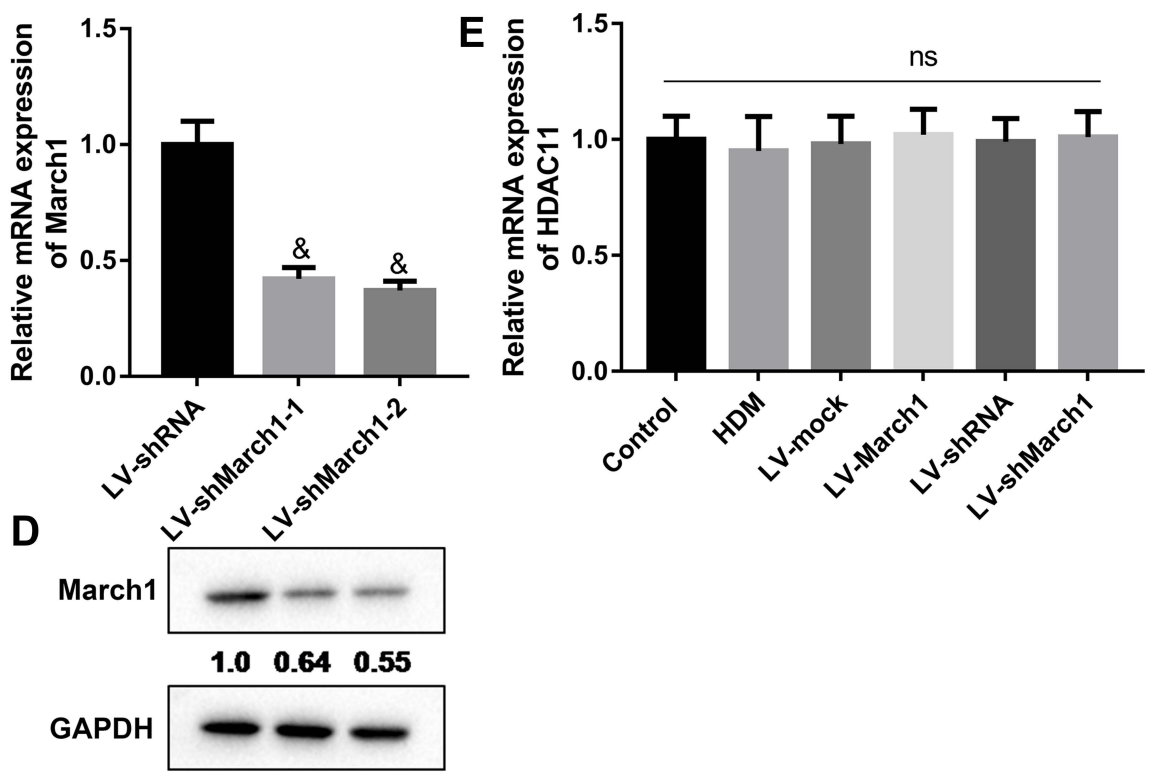

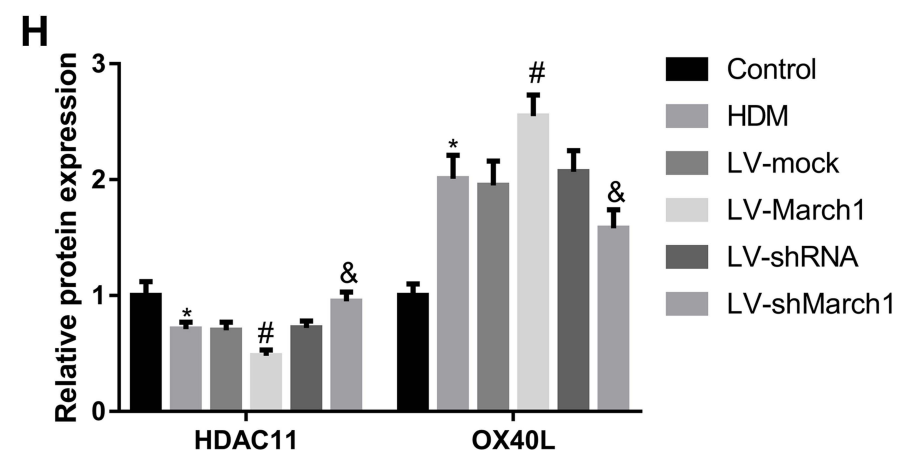

Figure 2 The regulation of March I on the expression of HDACII and OX40L. (A and B) DCs were infected with the lentivirus which carrying March I-expressing vector or empty vector. Subsequently, qRT-PCR and Western blot were performed to detect the expression of Marchl mRNA and protein, respectively, thus to test the infection efficiency. $n=3$. ${ }^{\#} P<0.0 .5$ compared with LV-mock group. (C and D) Two types of Marchl shRNA were designed, and were packaged into lentivirus to inhibit the expression of Marchl in DCs. qRT-PCR and Western blot were carried out to detect the expression of Marchl mRNA and protein, respectively. $n=3$. ${ }^{\&} P<0.05$ compared with LV-shRNA group. (E and F) DCs were infected with the lentivirus which carrying Marchl-expressing vector or MarchI shRNA, and qRT-PCR was carried out to examine the expression of HDACII and OX40L mRNAs. ( $G$ and $\mathbf{H})$ Western blot was performed to measure the expression of $\mathrm{HDACI}$ and $\mathrm{OX} 40 \mathrm{~L}$ proteins. $\mathrm{n}=3$. $* P<$ 0.05 compared with Control group, ${ }^{\#} P<0.05$ contrasted with HDM group, and ${ }^{\circledR} P<0.05$ contrasted with HDM group.

combination of March1 and HDAC11 in $293 \mathrm{~T}$ cells (Figure 4A) and DCs (Figure 4B). Our resulted also determined that March1 co-located with HDAC11 in intracellular (Figure 4C). As shown in Figure 4D, at 30 min after CHX treatment, a translation inhibitor, the protein level of
HDAC11 in the DCs infected with lentivirus-shRNA lower than that in the DCs infected with lentivirusshMarch1. Moreover, DCs were infected with the lentivirus expressing HDAC11 followed by $\mathrm{CHX}$ treatment. Our data indicated that the protein level of HDAC11 in 


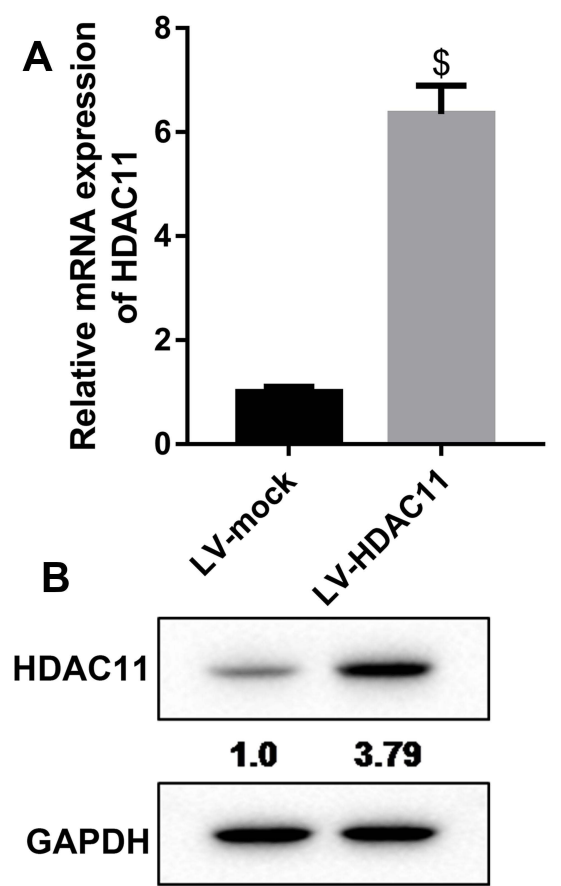

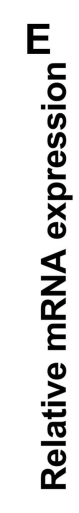

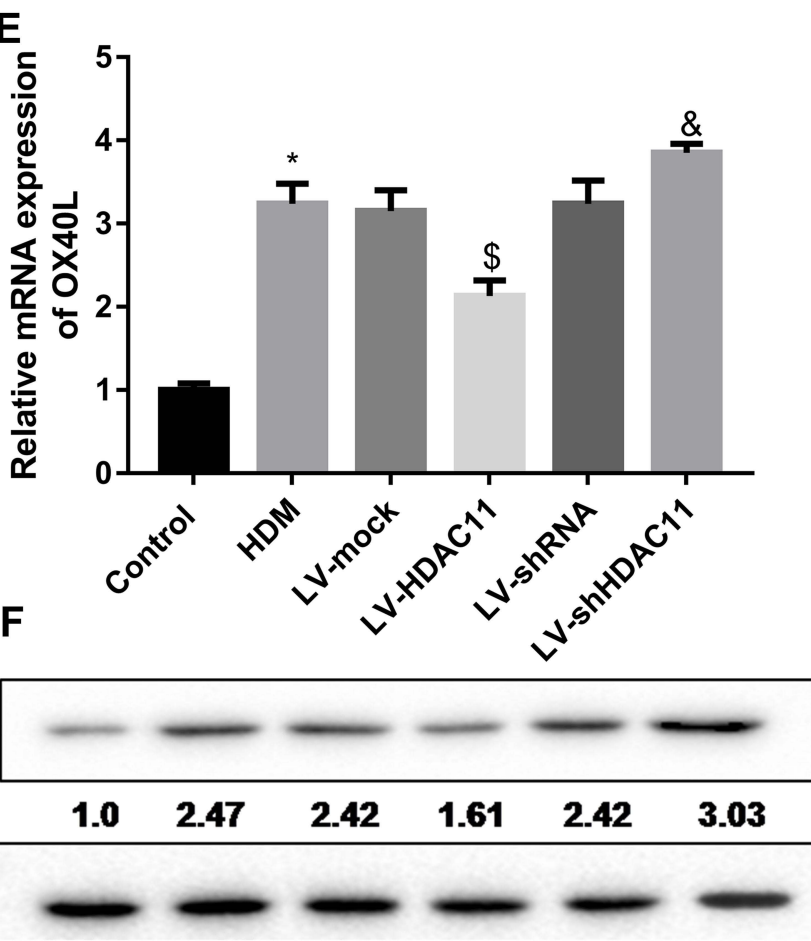

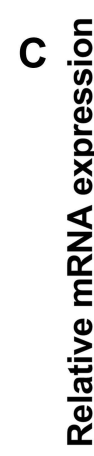

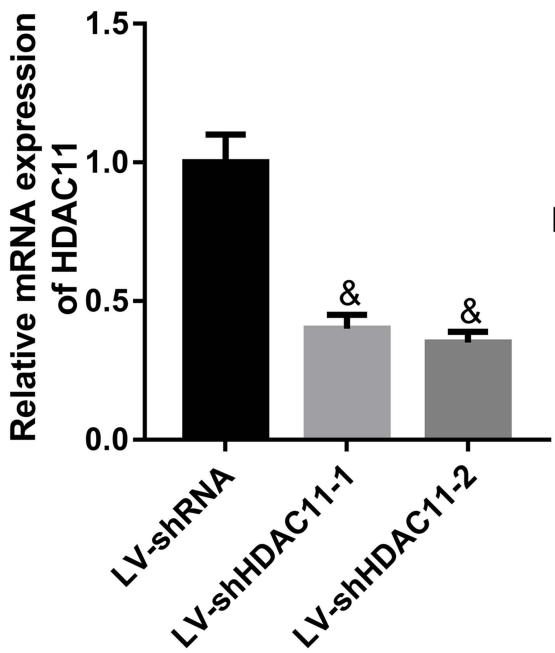

D

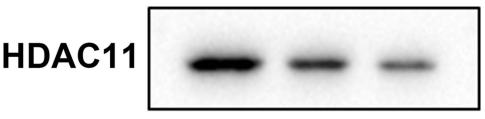

$1.0 \quad 0.50 \quad 0.46$

GAPD

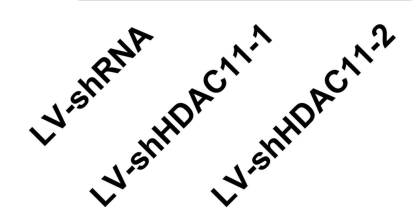

G

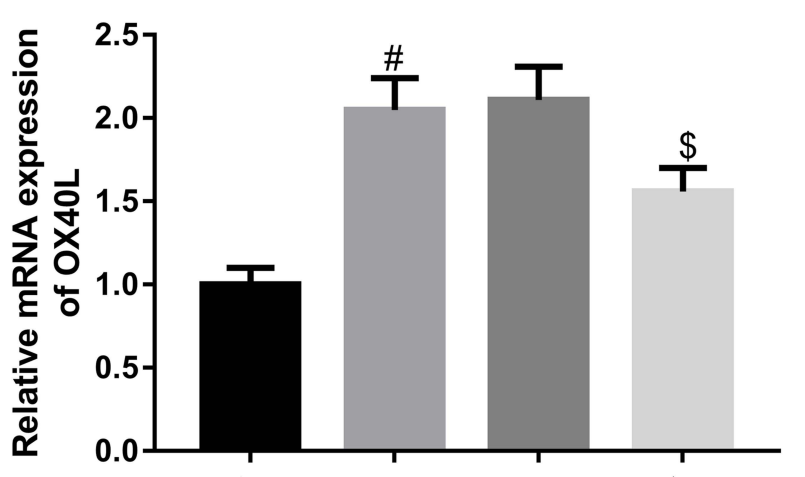

OX40L

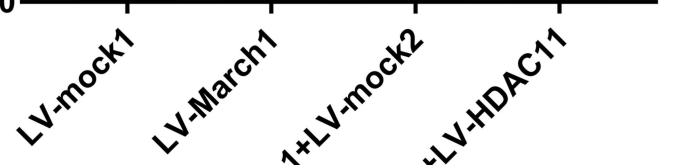

GAPDH

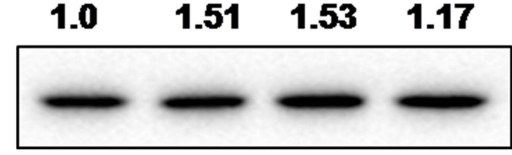

GAPDH

Figure 3 HDACII mediated the regulation of MarchI on OX40L expression in HDM-treated DCs. (A and B) DCs were infected with the lentivirus vector expressing HDACI I, and then the expression of HDACII mRNA and protein was measured by qRT-PCR and Western blot, respectively. $n=3$. ${ }^{\$} P<0.05$ compared with LV-mock group. (C and D) Two types of HDACII shRNA was designed. DCs were infected with the lentivirus vector carrying HDACII shRNA, and then the mRNA and protein levels of HDACII were carried out using qRT-PCR and Western blot. $n=3 .{ }^{8} P<0.05$ compared with LV-shRNA group. (E and F) DCs were infected with lentivirus vector to express HDACII or inhibit HDACI I expression followed by HDM stimulation. Then, the expression of OX40L mRNA and protein in the cells were measured by qRTPCR and Western blotting assay, respectively. $n=3$. $* P<0.05$ compared with Control group, ${ }^{\$} P<0.05$ compared with HDM group, and ${ }^{\&} P<0.05$ contrasted with HDM group. (G and $\mathbf{H}$ ) The HDM-treated DCs were co-infected with the lentivirus vector expressing March I and the lentivirus vector expressing HDACI I. Then, qRT-PCR and Western blot were carried out to examine the expression of OX40L mRNA and protein, respectively. $n=3$. ${ }^{\#} P<0.05$ compared with LV-mockl group, and ${ }^{\$} P<0.05$ contrasted with LV-March I group. 

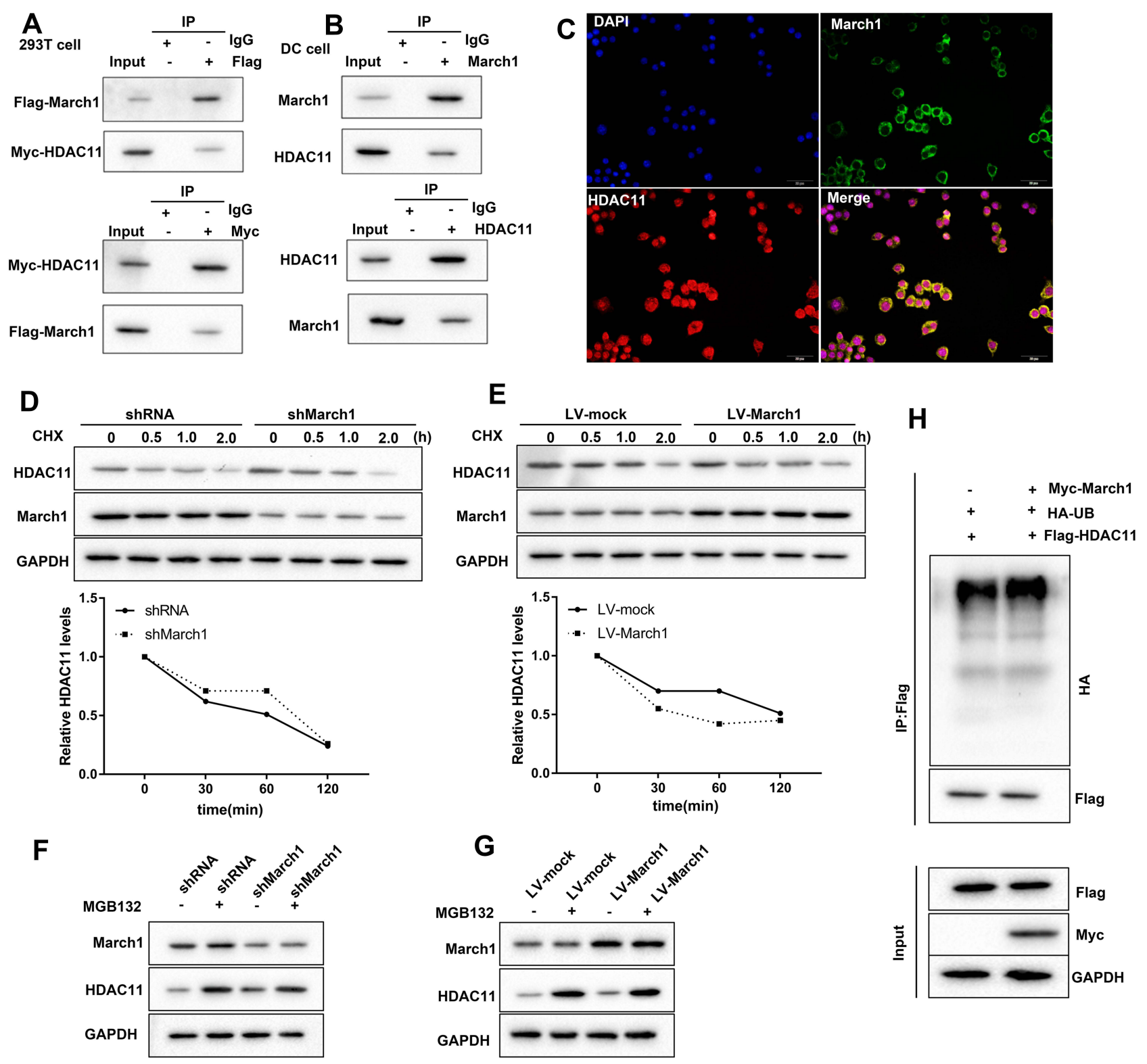

Figure 4 March I promoted HDACII ubiquitination and degradation. (A) Flag-March I and Myc-HDACII were co-transfected into HEK293T cells. Then, Co-IP assay was carried out to detect the combination of MarchI and HDACII. (B) Co-IP was performed to detect the combination of MarchI and HDACII in HDM-treated DCs. (C) Immunofluorescence staining March I and HDACII was performed to examine the intracellular localization of them. (D) DCs were infected with the lentivirus which carrying March I shRNA followed by CHX treatment, and then Western blot was carried out to detect the expression of HDACII and March I. (E) DCs were infected with the lentivirus expressing Marchl followed by CHX treatment. Then, the expression of MarchI and HDACII was measured by Western blot. (F) I0 $\mu g / m L$ of MGI32, a proteasome inhibitor, was used to treat DCs following the lentivirus infection. Western blot was performed to examine the expression of March I and HDACII. (G) I0 $\mu \mathrm{g} / \mathrm{mL}$ of MGI 32 was used to treat DCs following lentivirus infection. Then, Western blot was carried out to detect the expression of MarchI and HDACII in the cells. $(\mathbf{H})$ The vectors expressing Myc-March I, HA-UB, and Flag-HDACI I were transfected into DCs. Immunoblotting was used to analyze the level of HDACI I ubiquitination. $n=3$.

the DCs infected with LV-March1 was lower than that in the DCs infected with lentivirus-mock (Figure 4E). Silencing of March1 attenuated, while increasing of March1 enhanced the inhibition of CHX to HDAC11 synthesis. Our data also demonstrated that the expression of HDAC11 in DCs was significantly promoted by MG132 treatment, which was further increased by silencing of
March1 (Figure 4F). Whereas, the inhibitory effect of MG132 on HDAC11 degradation was partly reversed by increasing of March1 (Figure 4G). Importantly, overexpression of March1 obviously increased the level of HDAC11 ubiquitination (Figure 4H). Overall, March1 promoted the degradation of HDAC11 via inducing ubiquitination. 


\section{HDACI I Inhibited OX40L Expression Through the KLF-4-Dependent Pathway}

Furthermore, we investigated the molecular mechanism of HDAC11 decreasing OX40L expression. The interaction between KLF4 and HDAC11 was examined using reciprocal Co-IP assay with an anti-KLF4 and anti-HDAC11 antibodies. Our data showed that KLF4 interacted with HDAC11 (Figure 5A). In addition, the luciferase activity assay results indicated that overexpression of KLF4 significantly reduced the promoter activity of OX40L gene (Figure 5B), and silencing of KLF4 effectively enhanced the promoter activity of OX40L gene (Figure 5C). Increasing of HDAC11 also downregulated the promoter activity of OX40L gene, and decreasing of HDAC11 enhanced the promoter activity of OX40L gene (Figure 5D). Increasing of HDAC11 downregulated the mRNA and protein levels of OX40L in HDM-stimulated DCs, which was partly reversed by KLF silencing (Figure 5E and F). Summary, KLF4 mediated the inhibition of HDAC11 to OX40L.

\section{Discussion}

Asthma is an inflammatory disease. Indoor allergens, like HDMs, pets, rodents, cockroaches, and molds, are the major triggers of allergenic asthma. ${ }^{23}$ Previous studies confirmed that Th2 cells are a crucial meritorious factor for asthma development. However, the important role of DCs in the development of inflammation in asthma also cannot be underestimated. DCs are closely associated with innate immunity and adaptive immunity, and are the most potent antigen-presenting cells in immune system. ${ }^{24}$ Under the stimulation of HDMs and other risk factors, airway epithelial cells can secrete cytokines and other endogenous danger signals. It was reported that DCs could infiltrate to the position where close to airway epithelial cells, thus to enhance Th2 cells-mediated immunity response to allergen through promoting cytokines releasing from airway epithelial cells. ${ }^{25}$ In this study, we collected DCs from the serum of asthmatic children and matched healthy children, OVAinduced asthmatic mice and matched normal mice, and the bone marrow cells of normal mice. OVA stimulation and challenge is a common method to construct asthmatic model. ${ }^{26}$ Here, our results demonstrated that the mRNA and protein levels of March1 and OX40L were notably increased in the DCs from asthmatic children and asthmatic model. However, the expression of HDAC11 only in protein level was decreased in the DCs from asthmatic children and asthmatic model. Our data suggested that the abnormally expressed March 1 might participate in the development of asthma. Hence, we further investigate the molecular mechanism of March1 promoting OX40L expression in HDM-stimulated DCs.

OX40L, also named as CD252, is one of the members of TNFR/TNF superfamily. OX40L plays an important role in inflammatory response, and involves in the pathogenesis of many disorders, including asthma, through interaction with OX40. ${ }^{19,27}$ It was proved that inhibition the interaction of OX40L and OX40 using anti-OX40L $\mathrm{mAb}$ in an asthmatic mice could significantly attenuate the airway hyperresponsiveness of the mice and associated asthma symptoms on their. ${ }^{28} \mathrm{Li}$ et al reported that short ragweed pollen could trigger Th2 cellsmediated allergic inflammation through activating thymic stromal lymphopoietin (TSLP)/OX40L/OX40 signaling pathway. ${ }^{29}$ Damaged airway epithelial cells produce TSLP to stimulate the maturation of myeloid DCs, and then the activated DCs promote Th2 differentiation. The DCs expressing OX40L are an inducer of Th2 differentiation in asthma. ${ }^{30,31}$ OX $40 \mathrm{~L}$ affects the regulation of DCs to Th2 cells differentiation in asthma. Hence, we thought that exploring the expression mechanism of OX40L in DCs is very meaningful for understanding the pathogenesis of asthma. In this study, our results indicated that March1 was positively associated with the expression of OX40L in the HDM-stimulated DCs. Meanwhile, March1 negatively regulated the protein level of HDAC11, whereas has no effect on the level of HDAC11 mRNA.

March1 is an E3 ubiquitin ligase, and mediates the ubiquitination and degradation of target proteins. Ubiquitination has been ensured as a central event in the pathogenesis of almost all disorders, such as asthma. ${ }^{15}$ Previous study reported that March1 participates in the antigen presentation of DCs. ${ }^{32}$ Before our study, we have found that HDAC11 may be a substrate of March1 through the database of Ubibrowser (http://ubibrowser.ncpsb.org/ ubibrowser/). HDAC11 is a newest member of the HDAC family. HDAC11 not only has deacetylase activity but also has defatty-acylase activity. ${ }^{17}$ It was indicated that KLF4 could recruit HDAC11 to the promoter of target genes to regulate transcription, thus to response stimulation. ${ }^{33}$ Buglio et al proved that inhibition of HDAC11 could notably increase the expression of OX40L in Hodgkin lymphoma cell lines, thus to involve in immune response. ${ }^{34}$ Here, our data showed that March1 promoted 


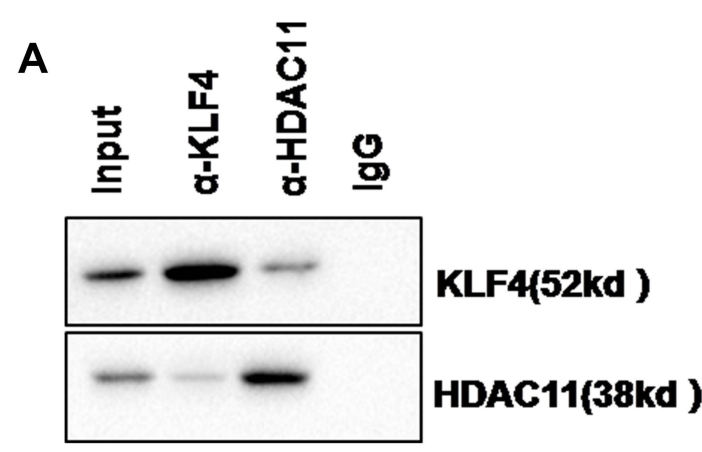

C
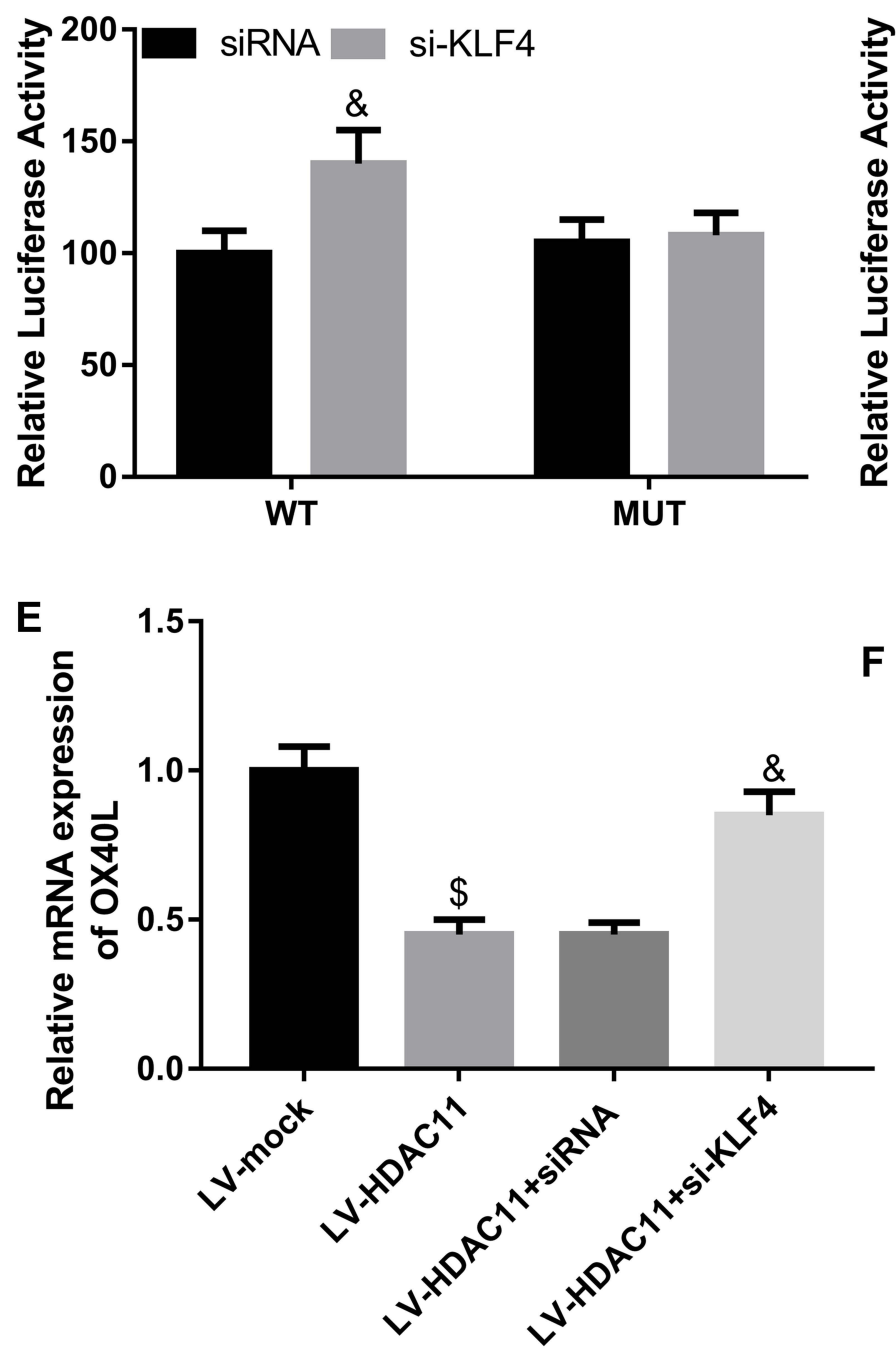

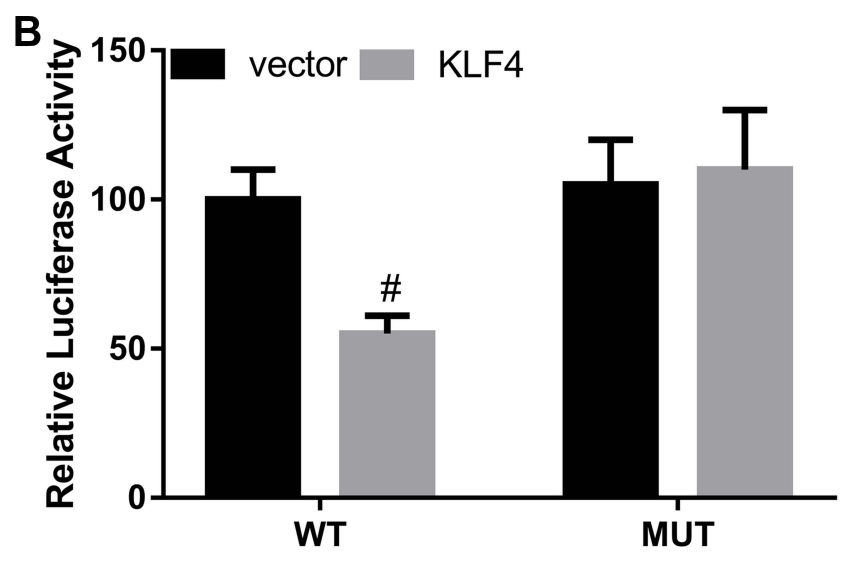

D

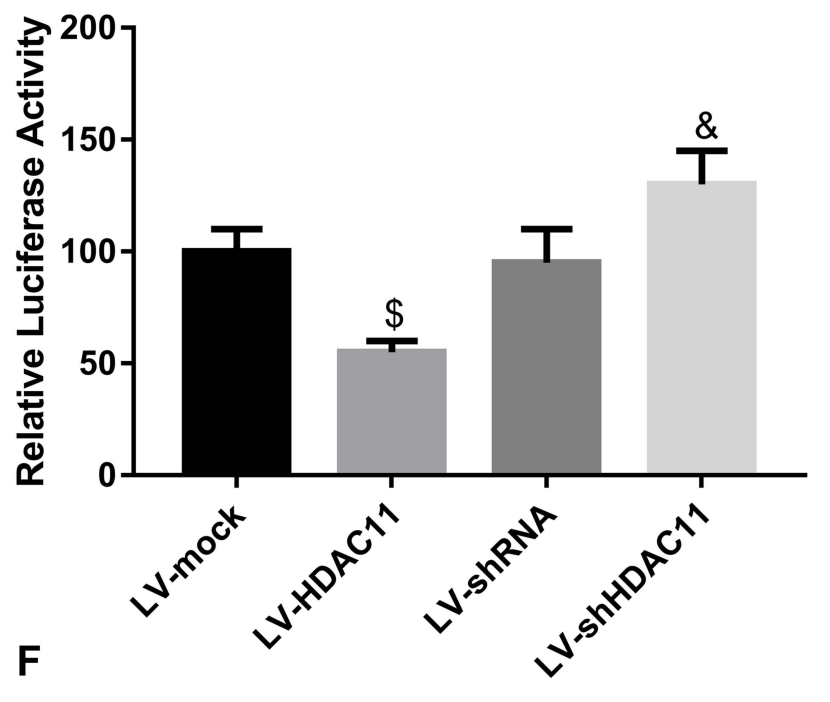

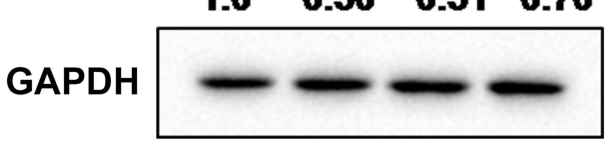

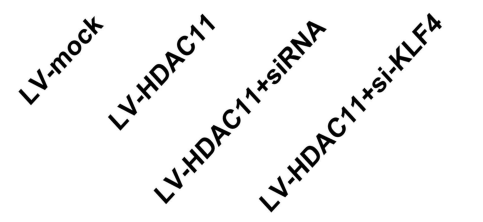

Figure 5 HDACII inhibited OX40L expression via KLF4-dependent way. (A) Co-IP was performed to examine the interaction between KLF4 and HDACII in HDMstimulated DCs. (B) The empty vector or pcDNA-KLF4 and the OX40L gene promoter-WT or OX40L gene promoter-MUT were co-transfected into HDM-stimulated DCs. Then, luciferase assay was carried out to detect the promoter activity of OX40L gene. ${ }^{\#} P<0.01$ compared with MUT + KLF4 group.(C) The siRNA negative control or KLF4 siRNA and the OX40L gene promoter-WT or OX40L gene promoter-MUT were co-transfected into HDM-stimulated DCs. Then, luciferase assay was carried out to detect the promoter activity of OX40L gene. (D) Luciferase assay was carried out to detect the promoter activity of OX40L gene in the HDM-treated DCs infected with the lentivirus vector expressing HDACII or carrying HDACII shRNA. (E and F) HDM-stimulated DCs were treated with the lentivirus vector expressing HDACII and the siRNA of KLF4. Then, $\mathrm{qRT}-\mathrm{PCR}$ and Western blot was carried out to detect the expression of OX40L mRNA and protein, respectively, in the cells. $\mathrm{n}=3$. ${ }^{\$} P<0.05$ compared with LV-mock group, and ${ }^{\&} P<0.05$ contrasted with LV-HDACI I group. 
the ubiquitination and degradation of HDAC11. HDAC11 negatively affected the expression of OX40L in HDMstimulated DCs. Furthermore, HDAC11 decreased the level of OX40L through reducing the promoter activity of OX40L via binding to KLF4.

\section{Conclusion}

Overall, our data demonstrated that March1 promoted OX40L expression through suppressing KLF4-dependent OX40L degradation by promoting the ubiquitination and degradation of HDAC11. Our data indicated a novel pathogenesis of asthma, and might provide a new idea or target for the treatment of the disease.

\section{Acknowledgments}

This study was supported by National Natural Science Foundation of China (No. 81971490, 81970027, 81771676); Suzhou Gusu Health Talents Program Stratified Training (Key Talents) (No. (2020)058); National Natural Science Youth Foundation of China (No. 81901260, 81900012).

\section{Disclosure}

All authors declare no conflict of interest.

\section{References}

1. Bonham CA, Patterson KC, Strek ME. Asthma outcomes and management during pregnancy. Chest. 2018;153(2):515-527. doi:10.1016/j.chest.2017.08.029

2. Rehman A, Amin F, Sadeeqa S. Prevalence of asthma and its management: a review. J Pak Med Assoc. 2018;68(12):1823-1827.

3. de Benedictis FM, Attanasi M. Asthma in childhood. Eur Respir Rev. 2016;25(139):41-47. doi:10.1183/16000617.0082-2015

4. Guilbert TW, Bacharier LB, Fitzpatrick AM. Severe asthma in children. $J$ Allergy Clin Immunol Pract. 2014;2(5):489-500. doi:10.1016/j.jaip.2014.06.022

5. Haktanir Abul M, Phipatanakul W. Severe asthma in children: evaluation and management. Allergol Int. 2019;68(2):150-157. doi:10.1016/j.alit.2018.11.007

6. Castillo JR, Peters SP, Busse WW. Asthma exacerbations: pathogenesis, prevention, and treatment. J Allergy Clin Immunol Pract. 2017;5 (4):918-927. doi:10.1016/j.jaip.2017.05.001

7. Patel SJ, Teach SJ. Asthma. Pediatr Rev. 2019;40(11):549-567. doi:10.1542/pir.2018-0282

8. Qian G, Jiang W, Zou B, et al. LPS inactivation by a host lipase allows lung epithelial cell sensitization for allergic asthma. $J$ Exp Med. 2018;215(9):2397-2412. doi:10.1084/jem.20172225

9. Casaro M, Souza VR, Oliveira FA, Ferreira CM. OVA-induced allergic airway inflammation mouse model. Methods Mol Biol. 2019;1916:297-301.

10. Mishra V, Banga J, Silveyra P. Oxidative stress and cellular pathways of asthma and inflammation: therapeutic strategies and pharmacological targets. Pharmacol Ther. 2018;181:169-182.

11. Malmström K, Lohi J, Sajantila A, et al. Immunohistology and remodeling in fatal pediatric and adolescent asthma. Respir Res. 2017;18(1):94. doi:10.1186/s12931-017-0575-0
12. Gon $\mathrm{Y}$, Hashimoto $\mathrm{S}$. Role of airway epithelial barrier dysfunction in pathogenesis of asthma. Allergol Int. 2018;67(1):12-17. doi:10.1016/ j.alit.2017.08.011

13. Qiu J, Sheedlo MJ, Yu K, et al. Ubiquitination independent of E1 and E2 enzymes by bacterial effectors. Nature. 2016;533(7601):120-124. doi:10.1038/nature 17657

14. Bourgeois-Daigneault MC, Pezeshki AM, Galbas T, et al. Tollipinduced down-regulation of MARCH1. Results Immunol. 2013;3:17-25. doi:10.1016/j.rinim.2013.02.002

15. Galbas T, Raymond M, Sabourin A, et al. MARCH1 E3 ubiquitin ligase dampens the innate inflammatory response by modulating monocyte functions in mice. J Immunol. 2017;198(2):852-861. doi:10.4049/jimmunol.1601168

16. Kishta OA, Sabourin A, Simon L, McGovern T. March1 E3 ubiquitin ligase modulates features of allergic asthma in an ovalbumin-induced mouse model of lung inflammation. $J$ Immunol Res. 2018;2018:3823910. doi:10.1155/2018/3823910

17. Liu SS, Wu F, Jin YM, Chang WQ, Xu TM. HDAC11: a rising star in epigenetics. Biomed Pharmacother. 2020;131:110607. doi:10.1016/j. biopha.2020.110607

18. Zhang HP, Wang L, Fu JJ, Fan T, Wang ZL, Wang G. Association between histone hyperacetylation status in memory $\mathrm{T}$ lymphocytes and allergen-induced eosinophilic airway inflammation. Respirology. 2016;21(5):850-857. doi:10.1111/resp.12774

19. Croft M, So T, Duan W, Soroosh P. The significance of OX40 and OX40L to T-cell biology and immune disease. Immunol Rev. 2009;229(1):173-191. doi:10.1111/j.1600-065X.2009.00766.x

20. Huang L, Wang M, Yan Y, et al. OX40L induces helper $T$ cell differentiation during cell immunity of asthma through PI3K/AKT and P38 MAPK signaling pathway. $J$ Transl Med. 2018;16(1):74. doi:10.1186/s12967-018-1436-4

21. Fernandez MV, Miller E, Krammer F, Gopal R, Greenbaum BD, Bhardwaj N. Ion efflux and influenza infection trigger NLRP3 inflammasome signaling in human dendritic cells. J Leukoc Biol. 2016;99(5):723-734. doi:10.1189/jlb.3A0614-313RRR

22. Wang S, Sun X, Zhou H, Zhu Z, Zhao W, Zhu C. Interleukin-4 affects the mature phenotype and function of rat bone marrow-derived dendritic cells. Mol Med Rep. 2015;12(1):233-237. doi: $10.3892 / \mathrm{mmr} .2015 .3349$

23. Gautier C, Charpin D. Environmental triggers and avoidance in the management of asthma. $J$ Asthma Allergy. 2017;10:47-56. doi:10.2147/JAA.S121276

24. Pan H, Zhang G, Nie H, Li S, He S, Yang J. Sulfatide-activated type II NKT cells suppress immunogenic maturation of lung dendritic cells in murine models of asthma. Am J Physiol Lung Cell Mol Physiol. 2019;317(5):L578-1590. doi:10.1152/ajplung.00256.2018

25. Plantinga $M$, Guilliams $M$, Vanheerswynghels $M$, et al. Conventional and monocyte-derived $\mathrm{CD} 11 \mathrm{~b}(+)$ dendritic cells initiate and maintain $\mathrm{T}$ helper 2 cell-mediated immunity to house dust mite allergen. Immunity. 2013;38(2):322-335. doi:10.1016/j.immuni.2012.10.016

26. Kim DI, Song MK, Lee K. Comparison of asthma phenotypes in OVA-induced mice challenged via inhaled and intranasal routes. BMC Pulm Med. 2019;19(1):241. doi:10.1186/s12890-019-1001-9

27. Salek-Ardakani S, Song J, Halteman BS, et al. OX40 (CD134) controls memory $\mathrm{T}$ helper 2 cells that drive lung inflammation. J Exp Med. 2003;198(2):315-324. doi:10.1084/jem.20021937

28. Kaur D, Brightling C. OX40/OX40 ligand interactions in T-cell regulation and asthma. Chest. 2012;141(2):494 499. doi:10.1378/chest.11-1730

29. Li DQ, Zhang L, Pflugfelder SC, et al. Short ragweed pollen triggers allergic inflammation through Toll-like receptor 4-dependent thymic stromal lymphopoietin/OX40 ligand/OX40 signaling pathways. J Allergy Clin Immunol. 2011;128(6):1318-1325.e1312. doi:10.1016/j.jaci.2011.06.041

30. Lee S, Shan J, Aldossary H, Gaudreault V, Bazett M, Fixman ED. STAT6 inhibitory peptide reduces dendritic cell migration to the lymph nodes to control Th2 adaptive immunity in the mouse lung. Eur J Immunol. 2019;49(1):157-169. doi:10.1002/eji.201847534 
31. Lin SC, Cheng FY, Liu JJ, Ye YL. Expression and regulation of thymic stromal lymphopoietin and thymic stromal lymphopoietin receptor heterocomplex in the innate-adaptive immunity of pediatric asthma. Int J Mol Sci. 2018;19(4):1231. doi:10.3390/ijms19041231

32. Baravalle G, Park H, McSweeney M, et al. Ubiquitination of CD86 is a key mechanism in regulating antigen presentation by dendritic cells. J Immunol. 2011;187(6):2966-2973. doi:10.4049/jimmunol.1101643
33. Zhou B, Zeng S, Li N, et al. Angiogenic factor with G patch and FHA domains 1 is a novel regulator of vascular injury. Arterioscler Thromb Vasc Biol. 2017;37(4):675-684. doi:10.1161/ATVBAHA.117.308992

34. Buglio D, Khaskhely NM, Voo KS, Martinez-Valdez H, Liu YJ, Younes A. HDAC11 plays an essential role in regulating OX40 ligand expression in Hodgkin lymphoma. Blood. 2011;117 (10):2910-2917. doi:10.1182/blood-2010-08-303701

\section{Publish your work in this journal}

The Journal of Asthma and Allergy is an international, peer-reviewed open-access journal publishing original research, reports, editorials and commentaries on the following topics: Asthma; Pulmonary physiology; Asthma related clinical health; Clinical immunology and the immunological basis of disease; Pharmacological interventions and

Submit your manuscript here: https://www.dovepress.com/journal-of-asthma-and-allergy-journal new therapies. The manuscript management system is completely online and includes a very quick and fair peer-review system, which is all easy to use. Visit http://www.dovepress.com/testimonials.php to read real quotes from published authors. 